\title{
Weighing communication overhead against travel time reduction in advanced traffic information systems
}

\author{
Rutger Claes • Tom Holvoet
}

Received: 25 July 2011 / Accepted: 8 February 2012 / Published online: 15 May 2012

(C) Springer-Verlag 2012

\begin{abstract}
Advanced traffic information systems can assist drivers in reducing their travel times by making better use of available road capacity. In assessing their practical applicability, however, it is important to assess the overhead that various advanced traffic information systems bring. This paper evaluates the communication overhead for a decentralized, delegate multi-agent system based advanced traffic information system and for a centralized system. We document the relationship between the communication overhead and travel time reduction for both systems. This analysis can help in weighing both factors when designing a practical traffic information system in a real-world scenario.
\end{abstract}

Keywords Advanced Traffic Information Systems . MultiAgent Systems · Traffic Guidance

\section{Introduction}

Rising traffic demand continues to fuel development of advanced traffic information systems, or ATISs, that improve the performance of the traffic networks. Performance of traffic networks can be improved by making better use of the network capacity and avoiding congestions. The implementation of ATISs results in a decrease of the total time spent by vehicles in the network.

The coordination of drivers in most ATISs depends on the sharing and spreading of information. Communication thus

\section{R. Claes ( $\varangle) \cdot$ T. Holvoet}

DistriNet Labs, Department of Computer Science,

Katholieke Universiteit Leuven, Celestijnenlaan 200A,

3001 Heverlee, Belgium

e-mail: rutger.claes@cs.kuleuven.be

T. Holvoet

e-mail: tom.holvoet@cs.kuleuven.be is key to the success of these systems. The overhead introduced by communication is a cost that has to be balanced with the benefits caused by the ATISs. The tradeoff thus created is hard to explore. Measuring the cost of an ATIS in a real-world setting is nearly impossible, as is measuring the benefits the system produces.

This paper explores the balance between communication overhead and decrease in vehicle travel times for two types of ATISs. The first ATIS is decentralized and uses link travel time predictions to guide vehicles, and the second ATIS is centralized and based on real-time information. Link travel time predictions and the benefits they can introduce, even at low penetration rates, are described by Wunderlich et al. [11]. Most link travel time prediction systems rely on information sent by the vehicles indicating their intentions and plans. The link travel time predictions used in the decentralized ATIS are generated using delegate multi-agent systems and online embedded simulations. These online simulations require information and thus communication. The generated link travel time predictions can lead to faster throughput times and shorter travel times for the vehicles participating in the system.

The second ATIS is a centralized system built around a central traffic information center, or TIC. This approach aims to limit communication overhead by providing vehicles with real-time values instead of predictions for the link travel times. By avoiding forecasts for link travel times, the centralized ATIS eliminates the need for knowing the vehicles' intentions. Real-time information is gathered from all roads in the network, but this causes less communication overhead. Both the decentralized and the centralized system are described more in-depth in, respectively, Sects. 2 and 3.

The benefits of both ATISs are evaluated by analyzing the sum and overall distribution of the travel time of all vehicles in the system. As the ATISs guide the vehicles more 
effectively, the travel time of the vehicles should decrease. By analyzing both the total travel time and the travel time distribution we include fairness in the evaluation.

The costs of both ATISs are evaluated by comparing the number of messages sent in both approaches. As the ATISs require more information exchange, the communication overhead will be higher. This paper focuses on the balance between the benefits and costs of information exchange and looks for tradeoffs in which the benefits are significant while the overhead is manageable.

To evaluate both ATISs, this paper uses a microscopic traffic simulation. The evaluation includes a simulation of both the traffic guided by the ATISs as well as the communication that is generated while guiding the traffic. Section 4 describes the experimental setup as well as the measures taken from the simulation used to evaluate both systems. Section 5 presents and analyses the results of the experiments.

The simulation used to evaluate both systems has certain limitations, namely a relatively simple communication model and a limited information about the simulated population. Both of these limitations place constraints on the measures used in the evaluation of the tradeoff. Analyzing the tradeoff between travel time reduction and message count can be considered as a first step towards better understanding the impact of decentralized ATISs. Real-world adoption of such complex systems will require evaluations based on more concrete real-world measurements such as bandwidth consumption and the economic benefits of the travel time reduction.

The paper concludes with an overview of related work in Sect. 6 and a conclusion in Sect. 7.

\section{Delegate multi-agent systems based ATIS}

The decentralized ATIS providing link travel time predictions is based on earlier work described in [2] and uses the concept of delegate Multi-Agent systems, introduced in [4] combined with online simulations, as presented in $[1,5]$. In this section we limit the description to an outline of the key principles of these techniques.

In this approach, vehicles are represented by vehicle agents and roadside infrastructure elements such as roads and junctions are represented by infrastructure agents. The vehicle agents are responsible for guiding their vehicle to its destination. They do this by calculating possible routes and evaluating these routes based on link travel time predictions. The infrastructure agents are responsible for providing link traversal time predictions. They generate these predictions by maintaining a model of the infrastructure element they represent and information about vehicles committed to traversing the infrastructure element. Both type of agents are geographically situated. They both represent an entity with an actual position in the real world.
Coordination in this decentralized system is achieved using delegate multi-agent systems [4]. Delegate multi-agent systems use lightweight agents to facilitate communication between agents. The lightweight agents are called ants because their behavior resembles that of real-world ants. These ants are mobile agents and move through the environment traveling from infrastructure element to infrastructure element, aggregating information, and communicating with infrastructure agents along their route. Delegate multiagent systems can perform certain complex tasks delegated to them by other agents. Vehicle agents, for example, delegate the exploration of the environment and maintenance of the information of their intentions in the environment. By delegating these tasks to separate multi-agent systems focussed solely on completing such a task, the complexity of the vehicle agents is greatly reduced.

Information about the vehicles' intentions is propagated by the vehicles using a delegate multi-agent system consisting of intention ants. These lightweight agents are dispatched by the vehicle agent at its current location. The intention ants then traverse the path the vehicle agent wants to use. Every infrastructure agent representing an infrastructure element on this path is notified of the pending visit. This notification includes the characteristics of the visiting vehicle and the time it intends to arrive. The notification is treated by the infrastructure agent as a pheromone, meaning the value of the information diminishes-or evaporates-over time. This evaporation has two consequences: (1) the vehicle agent has to resend intention ants at regular intervals; and (2) whenever a vehicle agent changes its intentions, it can disregard old notifications as they will evaporate automatically. The pheromone-like nature of the notifications thus ensures information about pending vehicle visits is kept up to date.

The information needed by the vehicle agents to decide on which route it intends to follow is gathered from the infrastructure elements using a second delegate multi-agent systems. The lightweight agents in this second delegate multi-agent system are called exploration ants and explore possible routes through the traffic environment. During their exploration of a possible route, the exploration ants interact with the infrastructure agents they encounter. As exploration ants traverse the environment, they keep track of the time it would take the vehicle they represent to reach their location. They use this horizon to ask infrastructure agents link travel time predictions at the time the vehicle they represent would pass the infrastructure element, represented by the current horizon. Using this prediction, they can update their horizon and progress to the next infrastructure element. On reaching their destination, the ants report back to the vehicle about the expected travel times if the vehicle were to choose this route.

To constrain the exploration ants on their exploration journey, the vehicle agent limits the number of possible routes they explore. The vehicle agent uses an $A^{*}$ based algorithm 
to select $K$ feasible paths based on its own beliefs about the link travel times of the network. The exploration ants explore these $K$ paths and report their findings back to the vehicle agent which can then update its beliefs about these paths. From this description, it is clear that the choice of $K$ greatly influences the communication overhead introduced by the exploration. The benefits of exploring more of the network as $K$ rises can lead to better performance. As vehicle agents have more information about potential routes, the options of avoiding congestion increase. Especially in traffic networks that offer many alternative routes to any destination.

\section{A central advanced traffic information system}

The decentralized ATIS described in the previous section is evaluated by comparing it with a centralized alternative. The centralized ATIS emphasizes low communication over performance and uses real-time data instead of link travel time predictions. Developing a centralized ATIS that can provide link travel time predictions is possible, as discussed in Sect. 6, but would require much more communication overhead.

The centralized ATIS implementation in this paper is a modification of the real-world traffic guidance based on the Traffic Messaging Channel (TMC). In the implementation described here, information is provided to the vehicles by a central traffic information center (TIC). The information is broadcasted to the vehicles using the TMC. Where technical limitations limit the set of roads on which vehicles receive information in the real world system, the implementation used here disregards these limitations and allows vehicles to request real-time information on all roads.

Vehicles can request real-time information about a number of routes through the traffic network. The TIC responds with real-time link travel times for all links in those routes. This additional information can be used by the vehicles to make better choices when faced with congestion and makes the guidance offered by the central system more competitive with that offered by the decentralized system previously described.

This adaptation implies the use of two way communication between TIC and vehicle, a requirement the TMC-based approach does not have. A second consequence of the adaptation is that the central TIC has to monitor all links in the network instead of just a limited set. The communication required by these adaptations, the two-way communication between vehicles and TIC, and the communication required to inform the TIC about the current state of the road, is also included in the evaluation.

Using the information provided by the centralized ATIS described above, vehicle agents can make informed decisions on what route to take to reach their destination. Allowing the vehicles to request real-time information about all links in the network would put too much stress on the communication. Vehicles are allowed to request information about $K$ routes. The TIC replies to such a request with the real-time link travel times for all $K$ routes.

Based on local information from either a static network description or previously requested information, the vehicle agent uses an $A^{*}$ based algorithm to construct $K$ feasible routes to its destination. This process is very similar to that used by the vehicle agent using the decentralized ATIS when deciding how to send out the exploration ants. The vehicle agent using the centralized ATIS requests the link travel times from the central TIC and merge the response into its beliefs about the network. It then calculates the best route to its destination using the $A^{*}$ algorithm and the newly updated information.

To avoid myopic behavior, the vehicle agents will hesitate before switching intentions. A newly discovered route has to be significantly better that the currently pursued route to switch intentions. Allowing myopic switching whenever a better route is discovered leads to instability and degrades performance.

Every minute, all roadside elements inform the central TIC about the conditions on their links. This communication is essential for the TIC to be able to relay this information to the vehicles. The implementation assumes no delays. Information is sent by the roadside elements to the central TIC and can be requested by the vehicle agents immediately. The updates sent by the roadside elements are unidirectional. The TIC never contacts a road. Every update thus counts for only one message.

\section{Experiment setup}

The experiments used to evaluate the ATISs described in the previous sections are based on simulations. This section will first describe the simulation used to obtain experimental results. Next, the experimental setup is described ${ }^{1}$. This includes how the vehicles are generated and how the ATISs are deployed in the simulated environment. Finally, we discuss and motivate our choice of measures.

The simulation environment models both the movement of the vehicles and the communication between agents deployed on vehicles or infrastructure elements.

Traffic in our evaluation is simulated on a microscopic level. Traffic is modeled in a multi-lane model. Traffic lights are not included in the simulation; all junctions have priority rules. The interaction between the ATISs and the simulation is shown in Fig. 1. The simulation model, including vehicles

\footnotetext{
${ }_{1}$ A more detailed description of the steps taken to set up the experiments can be found on http://people.cs.kuleuven.be/ rutger.claes/ papers/pai2012/index.html.
} 


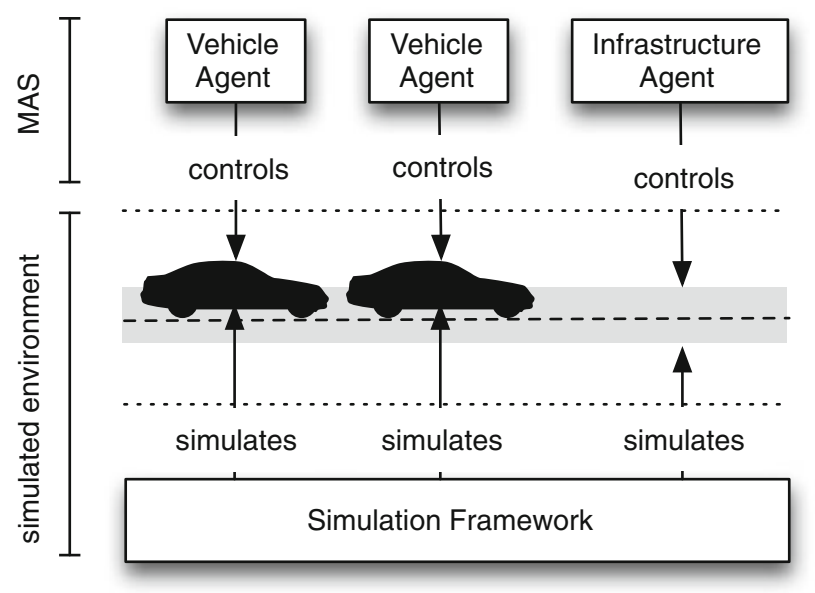

Fig. 1 Simulated environment and multi-agent system. The simulation simulates the different elements such as vehicles and infrastructure elements. Agents part of the ATISs control these simulated entities

and infrastructure elements, is simulated by our microsimulation framework. Agents taking part in the different ATISs control the simulated entities. Vehicle agents will coordinate the movements of the vehicles while infrastructure agents will forecast or collect and forward traffic information.

The simulated traffic environment is based on a real-world city. The evaluation takes place in and around the city of Leuven. When choosing the simulated environment for our evaluations a balance is sought between the scale of the simulated environment and the intensity of the traffic. In order to assess the benefits of our approach, a saturated road network is simulated. The desired traffic intensity on the roads severely limits the size of the simulated area. Simulating a medium-sized Belgian city allows us to simulate saturated traffic on a reasonable scale.

We have chosen the city of Leuven as the simulated environment out of pragmatic reasons. The parameterization of traffic and traffic network is a time-consuming effort. Because of previous experiments involving simulations of Leuven, we have already developed insights into traffic and routing patterns and were able to setup the experiments more quickly. Repeating these experiments on other maps, differing both in size and characteristics, remains necessary in order to better understand the properties of both ATISs evaluated in this paper.

Information about the simulated environment is extracted from OpenStreetMaps. OpenStreetMaps offers detailed information about road layout and characteristics. This allows the simulation to take into account the number of lanes, junction types, speed limits, and other information that affects traffic. The resulting traffic network contains over 1,600 links and 1,250 junctions, and is shown in Fig. 2.

Low-level driver behavior is modeled using a microscopic car following behavior [9], lane changing is done myopically.

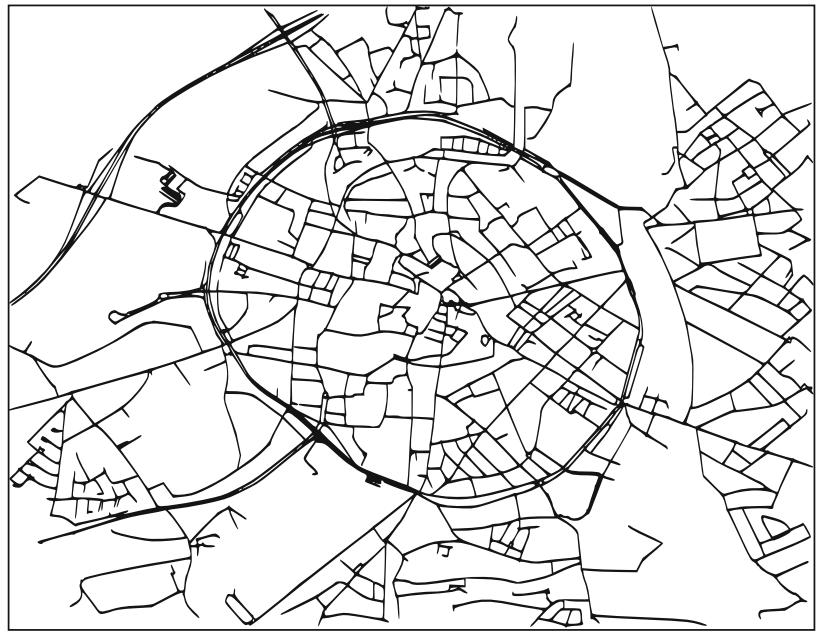

Fig. 2 The traffic graph created based on the OpenStreetMap data for the city of Leuven

The drivers' behavior is independent of the routing approach chosen. Vehicles guided by both approaches will react identically in traffic.

All simulations start using the same OD matrix describing the origin and destination of an artificial population of drivers. This OD matrix is constructed by randomly picking origins and destinations on opposite sides of the city center. The selection algorithm thus favors trips across the city. It is important to note that all simulations, regardless of the traffic saturation, the OD matrices and thus the vehicles, and their trips are identical.

Simulations use either the centralized or the decentralized ATIS to guide the vehicles to their destination. Four series of experiments are used: one using a centralized ATIS, referred to as tic and three other using a decentralized ATIS with different values for the $K$ parameter. The $K$ values are chosen to be 2,5, and 10. Empirical results indicate that a value of $K<5$ severely restricts the information obtained by vehicle agents, thus reducing the effectiveness of the ATIS. At values of $K>10$, performance no longer improves as the increase in information is no longer valuable. These empirical observations are highly dependent on the characteristics and size of the traffic network used in the simulation.

The simulations using the centralized ATIS use a value of $K=10$, the highest $K$ value used in the decentralized ATIS simulations. This ensures the vehicles in the tic simulations have knowledge of ten different routes, but receive this information by only sending and receiving one message. Increasing the $K$ value would only increase the size of the message, something the analysis disregards.

All four series of experiments consist of five simulations. In every simulation the traffic saturation is increased. Within each series of experiments describing an ATIS, traffic saturations of $20,40,60,80$, and $100 \%$ are simulated. 
The maximum saturation is determined empirically, but is identical for all four series. The saturation is increased without altering the origin or destination of vehicles. The vehicles described in the OD matrix are simply introduced into the network at a faster rate. Every simulation will have 18,000 vehicles.

\subsection{Used measures}

The simulation environment only monitors the number of messages sent; it currently disregards the message size and the path the message traverses to reach its endpoint.

For the decentralized ATIS based on delegate multi-agent systems, the message contents will contain only the list of infrastructure elements and the time the vehicle is expected to arrive at those elements. Every movement of an ant is counted as one message. The average number of elements included in a route will therefor influence the number of messages, as longer routes mean more ant movement. Because ants always travel between two adjacent infrastructure elements, the discarded routing overhead will be minimal.

In the centralized ATIS, every request for $K$ routes counts as one message, as does the reply sent by the central Traffic Information Center. The centralized ATIS thus benefits most from the routing and size simplification, as all roadside elements try to update the information at the central service and messages will generally travel much further.

Obtaining actual bandwidth measurements is currently not possible with the simulation model used in the evaluation. The metrics used are limited to message count. However, messages representing ant movements will not grow extremely large as they only contain a sequence of infrastructure element references mapped to estimated arrival times.

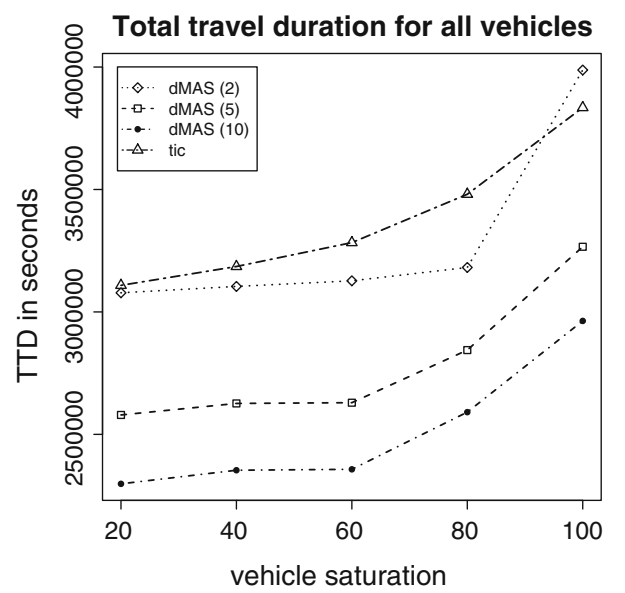

(a)
Improvement in traffic is measured by reduction in travel time. While the traffic network layout used in the simulations is based on real-world information, the simulated population is not. The economical consequences of the travel time reductions could be calculated if more in depth knowledge was available on the simulated population. In this paper, given the fact that the simulated population is generated artificially to achieve network saturation, no real world measures are used to quantify the benefits of a fictional driver population.

\section{Experiment results}

As previously described, the evaluation focusses on the number of messages exchanged between agents in both ATISs related to the performance obtained by reducing travel durations. First the travel durations for all previously described setups are shown and analyzed; next the messages needed to obtain these results are analyzed.

Figure 3 a depicts the total travel duration, i.e. the sum of all time spent by vehicles to reach their destination. Because of the increasing saturation of traffic, the time it takes all vehicles to reach their destination increases in both the experiments using the decentralized ATIS as in those using the centralized ATIS. Because the decentralized ATIS allows the vehicles to take into account predictive link travel times instead of real-time travel times, the total travel duration is less for the decentralized ATIS. The decentralized ATIS using delegate Multi-Agent Systems with a parameter $K$ of only two performs significantly worse than the other decentralized ATISs, especially at higher traffic saturations. As saturation increases, the limited amount of information received by the

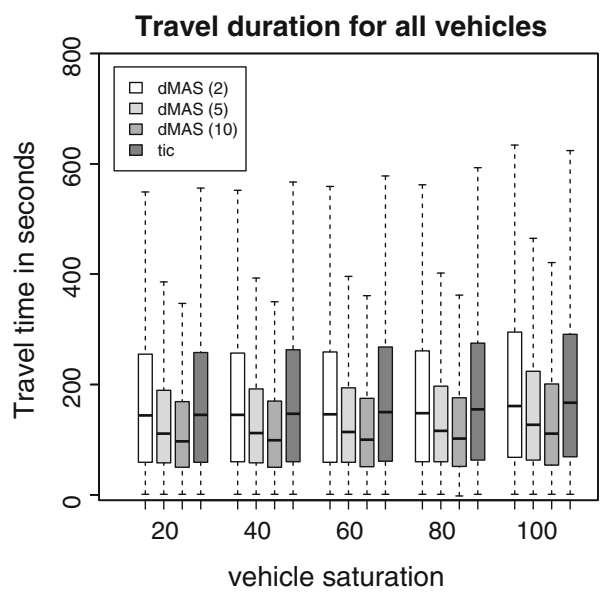

(b)

Fig. 3 Evolution of the travel duration for all vehicles as traffic saturation rises. $d M A S(K)$ denotes the decentralized delegate multi-agent based ATIS with parameter $K$. tic stands for the centralized ATIS. a Total travel duration, b travel duration distribution 


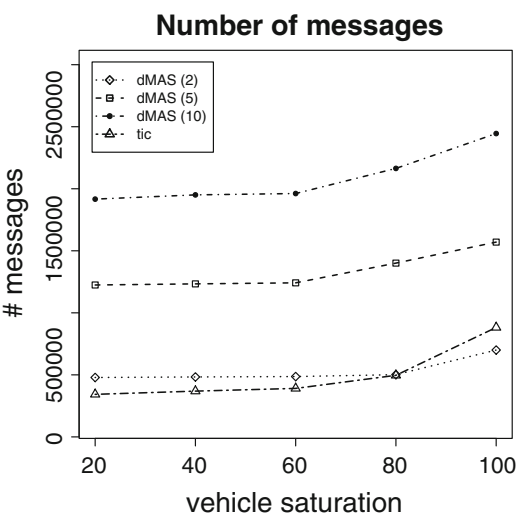

(a)

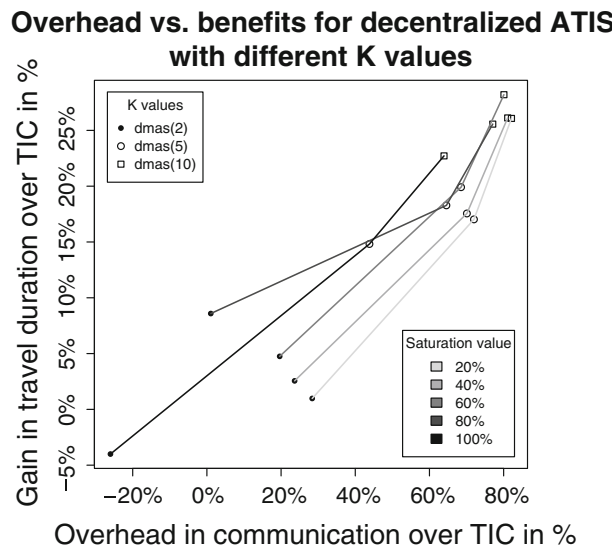

(b)

Fig. 4 a Evolution of the total number of message as traffic becomes more saturated. $\mathbf{b}$ Tradeoff between benefits and overhead for various values of $K$

vehicle agents no longer allows them to choose suitable congestion free routes.

These observations are confirmed by looking at the distribution of travel times for all vehicles in the system. Figure $3 \mathrm{~b}$ shows boxplots representing the entire population of vehicles' in every simulation. These boxplots show how the travel times are distributed. Each boxplot illustrates the minimum travel time at the bottom and the maximum travel time at the top. The box in the middle of each boxplot starts at the fist quartile and ends at the third quartile. The line dividing the box into two shows the median or second quartile value.

The boxplots in $3 \mathrm{~b}$ show that not only the mean, but also the third quartile and maximum value of the vehicle travel times is less when using decentralized ATISs with a parameter $K$ of at least five. The distribution of travel times is a measure of the fairness of the system, a criterium often neglected during evaluations.

The benefits of the decentralized ATIS and the high values for the $K$ parameter come at a cost. Figure 4a shows the total amount of messages passed between agents as the ATISs try to guide the vehicles. The line showing the total message count of the centralized ATIS includes the messages between infrastructure agents and the TIC. Even with these messages included, the number of messages sent in the decentralized ATISs is higher.

It is worth noting that with the centralized ATIS, around 100.000 of the messages are sent between the roadside infrastructure agents and the TIC. The rest of the messages are send between the TIC and the vehicle agents. All these messages are sent to or from one location, the TIC, thus creating a bottleneck.

Figure $4 \mathrm{~b}$ shows the tradeoff between the benefits and the overhead caused by decentralized ATISs for various values of the parameter $K$. As $K$ increases, the points move to the top right corner, indicating that the performance improves and the overhead increases. The lines show for each traffic saturation value how both benefits and overhead evolve as $K$ changes. If an acceptable communication overhead is known, Fig. 4b can be used to estimate a reasonable $K$ value.

The results presented in this section show that for all acceptable values of the exploration parameter $K$ the overhead introduced by the decentralized system is higher. When comparing communication in decentralized and centralized systems this is often to be expected. This paper, nevertheless, argues that a decentralized ATIS offers benefits to scalability and robustness. Even though the total message count in the decentralized ATIS is higher than that of the centralized ATIS, it spreads the communication overhead more evenly over the system. The centralized system, on the contrary, focusses all messages-along with most computationsin one central location. For ATISs, scalability and robustness clearly are requirements. These requirements suggest a decentralized approach. The bottlenecks and single point of failure introduced by the centralized system described in this paper threaten the scalability and robustness of the centralized approach.

Using delegate multi-agent systems to propagate and retrieve information from the distributed environment limits the communication overhead. If all distributed entities would communicate with each other directly, the communication overhead shown in Fig. 4a would be higher. The use of delegate multi-agent systems allows the vehicles to share their intentions only with interested infrastructure agents and to request information only from relevant infrastructure agents. Managing the audience that receives the information and keeping this audience as small as possible reduces the communication overhead.

The use of delegate multi-agent systems has a second benefit over direct communication. Because the ants are mobile 
agents, basically smart messages, they are capable of keeping track of the time horizon as they travel from network link to network link. Updating this horizon is necessary to request the correct link travel time prediction at the next infrastructure element. With direct communication, the vehicle agent would request information from an infrastructure element, wait for the response, calculate the horizon, and only then request information from the next infrastructure element. This would double the number of messages needed to obtain an estimate time of arrival for a route the vehicle agent is interested in. The lightweight ant agents take advantage of the path they have already traversed and the information they have accumulated when making the next request.

\section{Related work}

The benefits of link travel time predictions, such as those provided by the delegate MultiAgent systems approach, are described by Park and Rilett in [6] where they state that,

One of the major requirements of advance traveler information systems (ATISs) is a mechanism to estimate link travel times.

Work by Wunderlich et al. [11] has shown the benefits of the presence of link travel time predictions, even when the penetration rate of the participating vehicles is limited.

Link travel time predictions can be generated using a variety of different techniques such as online simulation [5], artificial neural networks [6], swarm computing [8], and machine learning [7]. A common requirement for all these approaches is communication. All approaches require information from roadside sensors or the intentions of vehicles to be aggregated and analyzed to predict link travel times.

Even though the benefits of link travel time forecasting are known and various link travel time predicting approaches are described in literature, research on the overhead these approaches introduce is scarce. Practical evaluations such as done by Van Der Horst and Nobel in [10], where they compare a decentralized to a centralized task allocation, are needed.

The work of Wunderlich et al. [11] shows the benefits of link travel time forecasts using a central service. The reduction of travel time for participating and non-participating vehicles is shown using a number of simulations. The effect of the penetration rate on the reduction of travel time is evaluated very thoroughly. The communication overhead introduced by the approach is not discussed; the results are limited to an evaluation of the benefits of the approach.

Work by Hunter et al. [5] describes the link travel time prediction using online simulation situated in the vehicles. Much attention is given to accuracy of the online simulations. The need for synchronization between the in vehicle simulations and protocols to achieve this synchronization are described. The communication requirements of such a vehicle to vehicle synchronization would be interesting when evaluating the feasibility and scalability of this interesting approach.

In [8], Tatomir et al. describe a mechanism called Ant Based Control that is very similar to the decentralized ATISs described in this paper. Ant-based control uses a combination of historical data and vehicle intentions to predict vehicle densities on the road. The relationship between speed and density is then used to predict the link travel time. The method of propagating and retrieving information used in [8] is very similar to that described in [2] and thus to the decentralized system described here, as both draw inspiration from Ant System described by Dorigo et al. in [3].

In their evaluation of decentralized and centralized task allocation systems in [10], the authors evaluate both systems by analyzing the robustness, performance, and energy consumption of both approaches. Their results can be used when deciding between a centralized and decentralized deployment. The methodology used by Van Der Horst and Nobel can be applied to many self-organizing systems where the benefits of using a decentralized over a centralized systems are unclear.

\section{Conclusion}

The evaluation presented in this paper illustrates the tradeoff between cost and benefit when looking at decentralized services. The delegate multi-agent systems approach presented in this paper allows the vehicle agents to take into account link travel time predictions and thus allows for faster travel times. But the increased communication between vehicles and roadside infrastructure comes at a cost.

Even though this paper evaluates a decentralized ATIS by comparing it with a centralized ATIS, the results indicate that the overhead produced by ATISs is also very sensitive to the implementation and configuration of the ATIS. Varying the exploration parameter $K$ greatly affects the communication overhead produced by the decentralized ATIS described in this paper. Besides analyzing the tradeoff between cost and benefits for centralized and decentralized approaches, it is also necessary to analyze the tradeoffs associated with parameters such as $K$. While the results are dependent on the concrete implementation of both the decentralized and centralized systems used, we believe that the order of magnitudes in both communication and travel time is inherent to the nature of the system and not its implementation.

The improvements in both scalability and robustness of a decentralized system have to be taken into account when evaluating these ATISs. By not relying on a single point of failure and avoiding the creation of bottlenecks, the decen- 
tralized system requires more communication. But since this communication is not targeted towards a single location, the required bandwidth for the individual communication links will most likely be manageable compared to the centralized ATIS.

Using delegate multi-agent systems to propagate and retrieve information is a way to control the audience when communicating. The use of smart messages behaving in an ant-like fashion ensures that information is only spread to relevant other parties. Because the ants can aggregate and analyze the information they obtain from the environment as they travel, less communication is required. Without the use of ants, vehicle agents would have to request information from the infrastructure agent, wait for a reply, calculate the estimated arrival time at the next link, and then request a link travel time forecast at the next link. Ants can calculate the estimated time of arrival at the next link and continue on their way.

Further evaluation of the use of delegate multi-agent systems remains a necessity. The conclusions drawn above are based on the simulation of only one street network. Studies on other maps, differing both in size and layout, should be conducted in further research to gain a more thorough understanding of the impact of distributed coordination mechanisms, such as the one presented in this paper, on communication and travel times. Quantifying the benefits and costs of both ATISs in more real-world measures such as economic cost and bandwidth utilization remains a necessary step before the impact of such systems can be fully understood.

Acknowledgments This research was funded by the IWT-SBO project 'MASE' (Project No. 060823) and by the Interuniversity Attraction Poles Programme Belgian State, Belgian Science Policy, and by the Research Fund K.U.Leuven.

\section{References}

1. Aydt, H., Turner, S., Cai, W., Low, M.: Symbiotic simulation systems: an extended definition motivated by symbiosis in biology. In: Proceedings of the 22nd Workshop on Principles of Advanced and Distributed Simulation, IEEE Computer Society, pp. 109-116 (2008)
2. Claes, R., Holvoet, T., Weyns, D.: A decentralized approach for anticipatory vehicle routing using delegate multi-agent systems. IEEE Trans. Intell. Transp. Syst. 12(2), 364-374 (2011)

3. Dorigo, M., Maniezzo, V., Colorni, A.: Ant system: optimization by a colony of cooperating agents. IEEE Trans. Syst. Man Cybern. Part B Cybern. 26(1), 29-41 (1996)

4. Holvoet, T., Valckenaers, P.: Exploiting the environment for coordinating agent intentions. Environ. Multi-Agent Syst. III:51-66 (2007)

5. Hunter, M., Sirichoke, J., Fujimoto, R., Huang, Y.L.: Embedded ad hoc distributed simulation for transportation system monitoring and control. In: Lee, L.H., Kuhl, M.E., Fowler, J.F., Robinson, S. (eds.) Proceedings of the 2009 INFORMS Simulation Society Research Workshop, vol. 1, pp. 13-17 (2009)

6. Park, D., Rilett, L.R.: Forecasting freeway link travel times with a multilayer feedforward neural network. Comput. Aided Civ. Infrastruct. Eng. 14(5), 357-367 (1999)

7. Roozemond, D.: Forecasting travel times based on actuated and historic data. Environment 23, 25 (1997)

8. Tatomir, B., Rothkrantz, L.J., Suson, A.C.: Travel time prediction for dynamic routing using ant based control. In: Rosetti, M., Hill, R.R., Johansson, B., Dunkin, A., Ingalls, R.G. (eds.) Proceedings of the 2009 Winter Simulation Conference, IEEE Computer Society, pp. 1069-1078 (2009)

9. Treiber, M., Hennecke, A., Helbing, D.: Congested traffic states in empirical observations and microscopic simulations. Phys. Rev. E 62(2), 1805-1824 (2000)

10. Van Der Horst, J., Noble, J.: Distributed and centralized task allocation: When and where to use them. In: IEEE International Conference on Self-Adaptive and Self-Organizing Systems Workshops (2010)

11. Wunderlich, K., Kaufman, D., Smith, R.: Link travel time prediction for decentralized route guidancearchitectures. IEEE Trans. Intell. Transp. Syst. 1(1), 4-14 (2000) 